University of South Florida

DIGITAL COMMONS

Digital Commons @ University of

@ UNIVERSITY OF SOUTH FLORIDA

South Florida

School of Geosciences Faculty and Staff

Publications

School of Geosciences

4-1990

\title{
The Effect of Tracking Network Configuration on GPS Baseline Estimates for the CASA UNO Experiment
}

\author{
S. Kornreich Wolf \\ California Institute of Technology \\ Timothy H. Dixon \\ California Institute of Technology, thd@usf.edu \\ J. T. Freymuller \\ University of South Carolina
}

Follow this and additional works at: https://digitalcommons.usf.edu/geo_facpub

Part of the Earth Sciences Commons

\section{Scholar Commons Citation}

Kornreich Wolf, S.; Dixon, Timothy H.; and Freymuller, J. T., "The Effect of Tracking Network Configuration on GPS Baseline Estimates for the CASA UNO Experiment" (1990). School of Geosciences Faculty and Staff Publications. 513.

https://digitalcommons.usf.edu/geo_facpub/513

This Article is brought to you for free and open access by the School of Geosciences at Digital Commons @ University of South Florida. It has been accepted for inclusion in School of Geosciences Faculty and Staff Publications by an authorized administrator of Digital Commons @ University of South Florida. For more information, please contact digitalcommons@usf.edu. 
GEOPHYSICAL RESEARCH LETTERS, VOL. 17, NO. 5, PAGES 647-650, APRIL 1990

\title{
THE EFFECT OF TRACKING NETWORK CONFIGURATION ON GPS BASELINE ESTIMATES FOR THE CASA UNO EXPERIMENT
}

\author{
S. Kornreich Wolf and T. H. Dixon \\ Jet Propulsion Laboratory, Califomia Instinute of Technology \\ J. T. Freymueller \\ Dept. of Geological Sciences, University of South Carolina
}

\begin{abstract}
Geodetic monitoring of subduction of the Nazca and Cocos plates is a goal of the CASA (Central and South America) Global Positioning System (GPS) experiments, and requires measurement of intersite distances (baselines) in excess of $500 \mathrm{~km}$. The major error source in these measurements is the uncertainty in the position of the GPS satellites at the time of observation. A key aspect of the first CASA experiment, CASA Uno,was the initiation of a global network of tracking stations to minimize these errors. We studied the effect of using various subsets of this global tracking network on long $(>100 \mathrm{~km})$ baseline estimates in the CASA region. Best results were obtained with a global tracking network consisting of three U.S. fiducial stations, two sites in the southwest Pacific and two sites in Europe. Relative to smaller subsets, this global network improved baseline repeatability, resolution of carrier phase cycle ambiguities, and formal errors of the orbit estimates. Describing baseline repeatability for horizontal components as $\sigma=\left(a^{2}+b^{2} L^{2}\right)^{1 / 2}$ where $L$ is baseline length, we obtained $a=$ 4 and $9 \mathrm{~mm}$ and $b=2.8 \times 10^{-8}$ and $2.3 \times 10^{-8}$ for north and east components, respectively, on CASA baselines up to $1000 \mathrm{~km}$ in length with this global network.
\end{abstract}

\section{Introduction}

The Global Positioning System (GPS) is becoming an important geodetic tool for monitoring plate motion and crustal deformation with centimeter-level precision. Some investigations require measurement of long $(>100 \mathrm{~km})$ baselines. Examples include study of relative displacement across oceanic spreading zones, diffuse continental transform zones, and plate convergence zones where at least one plate is oceanic and the only available measurement sites on that plate are islands, far from sites on the other plate. For the CASA (Central and South America) GPS experiments long-term scientific goals include monitoring spreading between the Nazca and Cocos plates, monitoring subduction of the Nazca and Cocos plates beneath Central and South America, and confirmation of and rates for compressional deformation along the diffuse southern Caribbean plate boundary (Kellogg et al., 1989). All of these goals require baselines well in excess of $500 \mathrm{~km}$. Geologic observations and global models predict plate rates of roughly $0-8 \mathrm{~cm} / \mathrm{yr}$ in the CASA region, with typical uncertainties of $\sim 0.5 \mathrm{~cm} / \mathrm{yr}$ (Mann and Burke, 1984; Stein et al., 1988). Assuming we desire a rate uncertainty $(1 \sigma)$ better than $0.3 \mathrm{~cm} / \mathrm{yr}$ with GPS experiments spanning 10 years, and assuming experiments every 2 years, individual measurements must achieve $1 \sigma$ position uncertainties of $\leq 2$ $\mathrm{cm}$, or $\leq 2$ parts in $10^{8}$ for a $1000 \mathrm{~km}$ baseline.

The major error source in measuring long baselines with GPS is the uncertainty in the position of the satellites at the

Copyright 1990 by the American Geophysical Union.

Paper number 90GL00420

$0094-8276 / 90 / 90 \mathrm{GL}-00420 \$ 03.00$ time of observation. A rough estimate of the sensitivity of baseline estimates to orbit errors is given by $\alpha_{\text {baseline }} \sim \alpha_{0} L / h$, where $\alpha_{\text {baseline }}$ is the error in the GPS baseline estimate due to orbit uncertainties, $c$ is a geometrical factor $(-0.2$; Lichten, $1990), \alpha_{0}$ is the GPS orbit error, $L$ is baseline length and $h$ is satellite altitude $(-20,000 \mathrm{~km})$. Typical uncertainties in the broadcast ephemerides of the satellites range from several meters to several tens of meters. Errors on estimates for baselines $>100 \mathrm{~km}$ may be unacceptably large $(>2 \mathrm{~cm})$ for the geological applications unless this effect is corrected.

The error in GPS baseline estimates due to orbit uncertainty can be reduced by performing simultaneous GPS observations from sites whose positions have been accurately determined to within a few centimeters by some other technique such as very long baseline interferometry (VLBI) (Dixon et al., 1985; Bock et al., 1986). The resulting fiducial network defines the reference frame for the GPS baselines of interest and provides data from which the satellite ephemerides can be estimated. Orbit uncertainties may be further reduced by acquiring tracking data from sites far outside the local network, and estimating the positions of those tracking sites which are not well known. An important aspect of the CASA Uno experiment was the initiation of a global network of tracking stations to minimize orbit-related errors on longer baselines.

We investigate the effect of using subsets of this global tracking network on the repeatability of long baseline estimates in the CASA region using data from the CASA Uno experiment. We compare results obtained with a U.S.-only fiducial network, to results obtained when this network is supplemented by tracking stations in the southwest Pacific and Europe.

\section{Data Analysis}

GPS results were obtained using the GIPSY (GPS Inferred Positioning SYstem) software developed at the Jet Propulsion Laboratory (Lichten and Border, 1987; Sovers and Border, 1987). Estimated parameters include satellite positions and velocities, station positions, carrier phase ambiguities, satellite and station clocks and zenith tropospheric path delays. Satellite and station clocks were modeled as white noise, and combined wet and dry zenith tropospheric path delay was modeled as a stochastic process (Tralli et al., 1988). Both dual frequency carrier phase and $P$-code pseudorange data were included in the analysis. Carrier phase cycle ambiguities were resolved using the method of Blewitt (1989). Only single-day arcs were processed. Each station in the CASA region observed for 7.5 to 9 hours daily.

In order to ensure consistent fiducial geometry from day to day, three stations that were generally available throughout the experiment were chosen for the United States fiducial network: Mojave, CA; Ft. Davis, TX; and Westford, MA (Table 1). Their relative positions have been established by VLBI (solution GLB223, J.W. Ryan, C. Ma and E. Himwich, written communication) combined with local GPS ties, and are considered known at the centimeter-level. The positions of these three sites were held fixed in the analysis. In addition to these U.S. sites, a core of five CASA sites were chosen to 
TABLE 1. GPS Data Availability for the CASA Uno Experiment*

\begin{tabular}{|c|c|c|c|c|c|c|c|c|}
\hline$\overline{\text { STATIONS }}$ & JAN 19 & 20 & 21 & 22 & 25 & 26 & 27 & 29 \\
\hline Ft. Davis (U.S.) & 0 & 0 & 0 & $\overline{0}$ & 0 & 0 & 0 & 0 \\
\hline Haysiack & 0 & 0 & 0 & 0 & 0 & 0 & 0 & 0 \\
\hline Mojave & 0 & 0 & 0 & $x$ & 0 & 0 & 0 & 0 \\
\hline OVRO & $x$ & $x$ & $\mathrm{X}$ & 0 & $\mathbf{X}$ & $\mathrm{X}$ & $\mathbf{X}$ & $\mathbf{x}$ \\
\hline Albrook (Panama) & 0 & 0 & 0 & 0 & 0 & 0 & 0 & 0 \\
\hline Liberia (Costa Rica) & 0 & 0 & 0 & 0 & 0 & 0 & $\mathbf{X}$ & 0 \\
\hline Limon & 0 & 0 & 0 & $x$ & 0 & 0 & 0 & 0 \\
\hline Cocos Island & $x$ & 0 & 0 & 0 & 0 & 0 & 0 & 0 \\
\hline Bogora (Colombia) & $x$ & $x$ & $x$ & $\mathrm{x}$ & 0 & 0 & $\mathrm{O}$ & 0 \\
\hline $\mathrm{Cali}$ & $\mathrm{O}$ & 0 & 0 & 0 & 0 & 0 & 0 & 0 \\
\hline Malpelo & 0 & 0 & 0 & 0 & 0 & 0 & 0 & 0 \\
\hline Moco2 & 0 & 0 & 0 & 0 & $x$ & $x$ & $x$ & $\mathrm{X}$ \\
\hline Pasto & 0 & 0 & 0 & 0 & $\mathbf{X}$ & $\mathrm{X}$ & $\mathbf{X}$ & $\mathbf{X}$ \\
\hline Tumaco & 0 & $x$ & $\mathbf{x}$ & 0 & $\mathbf{x}$ & $\mathrm{x}$ & 0 & $\mathrm{X}$ \\
\hline Villavicencio & $\mathbf{x}$ & $x$ & $\mathbf{x}$ & $\mathrm{x}$ & 0 & 0 & 0 & 0 \\
\hline Onsala (Sweden) & 0 & 0 & 0 & 0 & 0 & 0 & 0 & 0 \\
\hline Wettzell (FRG) & 0 & 0 & 0 & 0 & 0 & 0 & 0 & 0 \\
\hline Canberra (Aus) & 0 & 0 & 0 & 0 & 0 & 0 & 0 & 0 \\
\hline Blackbirch (N.Z.) & 0 & 0 & 0 & 0 & 0 & 0 & 0 & 0 \\
\hline
\end{tabular}

* o Data available and used $\mathrm{X}$ Data not available or not used

ensure consistent regional geomerry: Malpelo, Columbia; Cocos Island, Limon and Liberia, Costa Rica; and Albrook, Panama. The core regional stations were also available during most of the experiment with a few exceptions (Table 1). Additional regional stations were included so that a total of eight stations was available each diy in Central and South America. The positions of these eight stations were estimated in the analysis with a loose a priori location constraint of 2 $\mathrm{km}$. Eight out of fifteen days of data met these availability criteria and were used in this analysis (Table 1).

Three tracking networks were compared. Network (A) consists of the three fixed stations in the United States. Network (B) consists of network (A) plus two stations located in the southwest Pacific at Canberra, Australia and Black Birch, New Zealand. Network (C) consists of network (B) plus two European sites: Onsala, Sweden and Wettzell, West Germany. The European and Australian sites are, like the U.S. sites, collocated with VLBI monuments. However, initial analysis showed changes in station position at the European and Australian sites on the order of tens of centimeters relative to a priori position values (also determined from GLB223 and local site ties). It is possible that these changes were due to errors in the ties from the VLBI markers to the GPS monuments since such measurements can be difficult to make. Thus, these station positions were not held fixed but rather were conservatively estimated with a priori spatial constraints of 1 meter. In the subsequent discussion, the term "global tracking network" refers to the set of seven sites consisting of the fixed U.S. sites and the estimated European and southwest Pacific sites

\section{Results and Discussion}

The day-to-day scatter of daily estimates about the mean (short-term repeatability) is a measure of GPS performance, and can be used to test the effect of tracking network configuration. Tracking network configuration strongly affected the repeatability of horizontal baseline components, but did not affect repeatabilty of vertical baseline components. Mean vertical repeatabilities for nethorks (A), (B) and (C) are $5.4,5.7$ and $5.7 \mathrm{~cm}$, respectively. This is probably due to the fact that errors in GPS-derived vertical component estimates are dominated by atmospheric effects, similar to VLBI (Herring, 1986).

It is useful to consider the precision, or repeatability, of a horizontal component of a GPS baseline estimate, $\sigma$, in terms of a baseline length-independent term, a, which includes instrumental effects, and a baseline length-dependent term, $b$, dominated by errors associated with satellite orbit uncertainties. We assume that horizontal baseline component uncertainties have the following functional form:

$$
\sigma=\left(a^{2}+b^{2} I^{2}\right)^{1 / 2}
$$

where $I$ is baseline length. For a typical GPS experiment, a is $<1 \mathrm{~cm}$, while b may range from $1 \times 10^{-8}$ to $1 \times 10^{-6}$ depending on experiment design and analytical techniques. Our experiment spans a large range of baseline lengths, but has few baselines $<100 \mathrm{~km}$. Thus, our data do not constrain a as well as experiments with numerous short baselines (e.g., Davis et al., 1989). However, b is well determined by our experiment, and the effect of different tracking network configurations on baseline precision can be investigated by looking at variations in b. GPS baseline estimates in southem California, where GPS visibility is strong, wet tropospheric path delay errors are small (Tralli et al., 1988) and numerous VLBI sites are available for orbit tracking and baseline accuracy assessment, have achieved precision with a lengthdependent term (similar to $\mathrm{b}$ in equation 1) of $1-2 \times 10^{-8} \mathrm{for}$ horizontal components (Dong and Bock, 1989; Blewitt, 1989, Davis et al., 1989). An optimal global tracking network for the CASA Uno experiment could, therefore, approach this level of baseline precision, although errors associated with wet tropospheric path delay may be higher in the CASA region (Dixon and Kornreich Wolf, this issue).

Figure 1 shows repeatability for horizontal components for 3-8 days of available data for the 17 Central and South American baselines defined by our regional network with lengths of $<1000 \mathrm{~km}$ for networks (A), (B) and (C). Also shown are best fit curves of the form described by equation 1 together with corresponding values for $a$ and $b$. As the tracking network is augmented with additional stations, $b$ is improved for both the north and east baseline components Note that the variation in a from $0-11) \mathrm{mm}$ is an artifact of the scatter in the data and is not meaningful for determining GPS error at short baseline length. Other studies show that GPS position errors for baselines $<20 \mathrm{~km}$ are in the range $2-5 \mathrm{~mm}$ (Davis et al., 1989; Dong and Bock, 1989). Because of possible correlation between $a$ and $b$ in the least squares fitting, we also fixed $a=5 \mathrm{~mm}$ and varied $b$ only. Resuling values for $b$ were not significantly different from the values shown in Figure 1.

Based on the value of $b$ obtained from the least squares fit to the repeatability data, we can infer that addition of data from Australia and New Zealand to the U.S.-only network (A) significantly improves both east and porth componeat estimates for the longer baselines. East componem repeatability improves by nearly a factor of two with the addition of these southwest Pacific sites to network (A), while north component repeatability improves by a factor of 1.4 When the two European sites are added to network $(B)$, additional factor of 1.4 improvement is achieved in component repeatability, but no improvement is seen in nort component repeatability. We also performed analyses shown in figure 1) with network (A) plus the two Europen sites only; although there were small improvements in $\mathrm{b}$, w inclusion of the two southwest Pacific sites to network (A proved almost twice as effective. The relatively sos improvement with addition of data from Europe compared to the significant improvement with addition of data from the southwest Pacific may be due to two factors. With the curs GPS satellite configuration, there are four areas of the glot (the U.S., China, south Atlantic and southwest Pacific) the satellites converge, enabling simyltaneous observation of greater number of satellites in these regions relative to Europ Also, the southwest Pacific stations expand both the N-S E-W extent of the tracking network significantly, wherf addition of data from the European sites to a three station $\mathrm{Uh}$ 


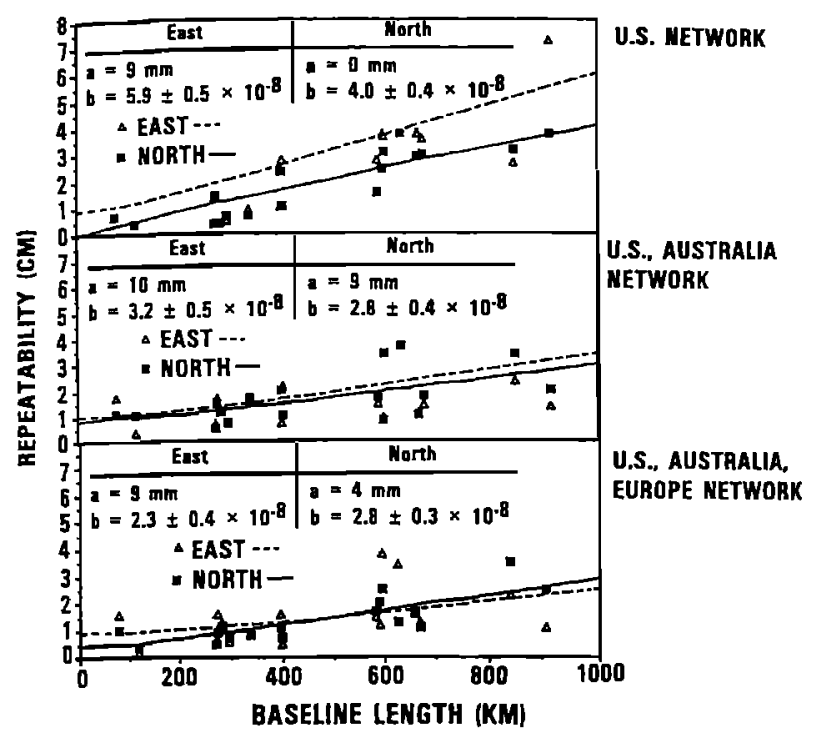

Fig. 1. East and north repeatabilities based on 3-8 days of data depending on station availability plotted versus baseline length for 17 baselines in the Central and South America (CASA) region. Best fit curves (weighted by the average of the formal errors of the baseline component estimates divided by the square root of the number of days of data) and corresponding $a$ and $b$ values from equation 1 are also shown.

network only expands the E-W extent of the tracking network, and by a relatively smaller amount.

Although addition of data from the Australian and New Zealand sites has the most significant effect on the repeatability of the horizontal components, the strongest network involves all seven global tracking sites. The use of global tracking network (C) allows a factor of 2.6 and 1.4 improvement on the east and north component repeatability, respectively, as compared to a U.S.-only network (A). Global tracking network (C) yields baseline repeatability for east and north components, as represented by $b$ in equation 1 , of $2.3 \times 10^{-8}$ and $2.8 \times 10^{-8}$ respectively on CASA baselines.

The formal errors of the horizontal baseline component estimates improve by a relatively small amount ( factor of 1.4) compared to the -factor of 2.6 improvement in repeatability from network (A) to network (C). Perhaps the main effect of the global tracking network approach is to reduce systematic errors associated with fiducial station data and resulting orbit estimates.

It is not possible at this time to determine the accuracy of the CASA region GPS baseline estimates because alternative techniques are not available for comparison. However, we believe that it is possible to demonstrate that tracking network configuration affects the accuracy of the baseline estimates. It has been shown that resolving canier phase cycle ambiguities ("bias fixing") improves GPS baseline accuracy by factors of 2-3 (Blewitt, 1989; Dong and Bock, 1989). The ability to resolve carrier phase cycle ambiguities thus provides an additional method of assessing the utility of various network configurations and indirectly indicates baseline accuracy improvement. Note that we attempt to resolve only the cycle ambiguities associated with the local Central and South America network, i.e., exactly the same set of biases are analyzed for each network. Figure 2 shows three lines of evidence suggesting that network (C) improves baseline accuracy relative to the two smaller networks, because of effects related to cycle ambiguity resolution. First, the number of cycle ambiguity estimates before bias fixing with formal errors $<0.2$ cycles from all 8 days of data vary with network configuration; network (C) yields the greatest number of bias estimates with formal errors less than 0.2 cycles. Second, the
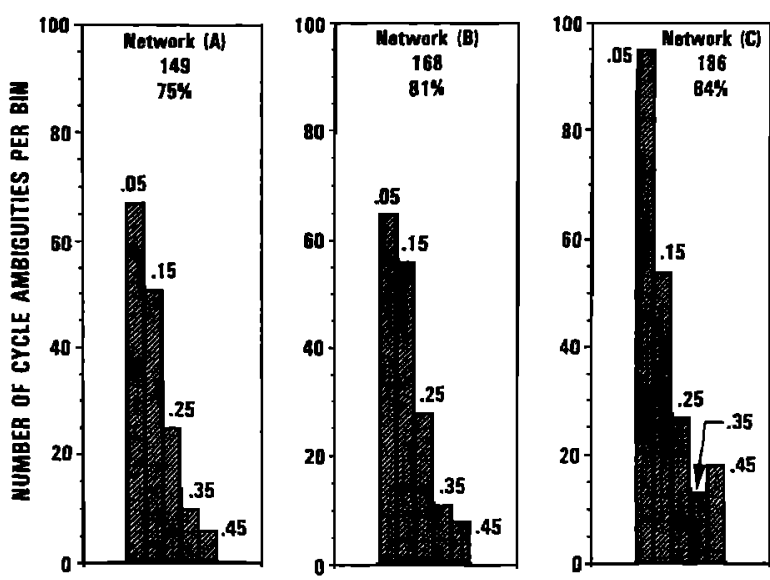

\section{CYCLES FROM NEAREST INTEGER}

Fig. 2. Bias estimates with formal errors $<0.2$ cycles from 8 days of data for only the 8 stations in Central and South America used in this analysis. Each bin shows the distance, in fractional cycles, between the estimate and its nearest integer value. The vertical axis shows the number of biases, with formal errors $<0.2$ cycles, that fall into each bin for all 8 days. The first value above each graph shows the total number of bias estimates with formal errors $<0.2$ cycles. The second value shows the number of biases fixed to their closest integer value for each network (with a confisence level of $99 \%$ ) as a percentage of all biases associated with the local CASA network.

distribution of bias estimates close to integer cycles is affected by network configuration; the bias estimates from network (C) have a distribution that is closer to integer cycles than the two smaller networks. Ideally, a highly peaked, half Gaussian shape is obtained in graphs such as Figure 2, representing a probabilistic model for the range of accuracy of the bias estimates. Third, the percentage of cycle ambiguities resolved is affected by network configuration; $9 \%$ more carrier phase ambiguities are resolved with network (C) relative to network (A), presumably yielding more accurate baseline estimates.

We attribute the improvement in baseline precision (inferred from improvements in repeatability) and accuracy (inferred on the basis of increased cycle ambiguity resolution) to the increased geographic extent of the tracking network, giving stronger observation geometry, and to consequent improvements in the satellite orbit estimates. It is possible to quantify the improvement in orbit estimates by examining the orbit uncertainties of several satellites for the three tracking networks. One method of assessing the improvement in orbit determination for the various networks is illustrated in Figure 3 where the root mean square (rms) of the formal errors of the three orbit components for four representative satellites is propagated in time for a single-day arc. This figure shows that significant improvement in the formal errors of the orbit estimates is obtained when a stronger tracking network is used. Network (C) sustains three-dimensional position uncertainties for the four satellites of 1-5 meters over the entire 24 hour observation period, whereas networks (A) and (B) show large fluctuations, in the 1-15 meter range over the same 24 hour period. The minimum orbit errors in the middle of each graph correspond to the period of several hours when the satellite constellation converges over the U.S. During this period, orbit uncertainties for all four networks are similar. Outside this period, however, the orbit estimate degradation for networks (A) and (B) is severe, suggesting that the orbital states determined from the global tracking network (C) are more precise. Because the baseline components are highly correlated with GPS orbital states, a corresponding improvement in regional baseline precision can be expected, particularly for the longer baselines. 

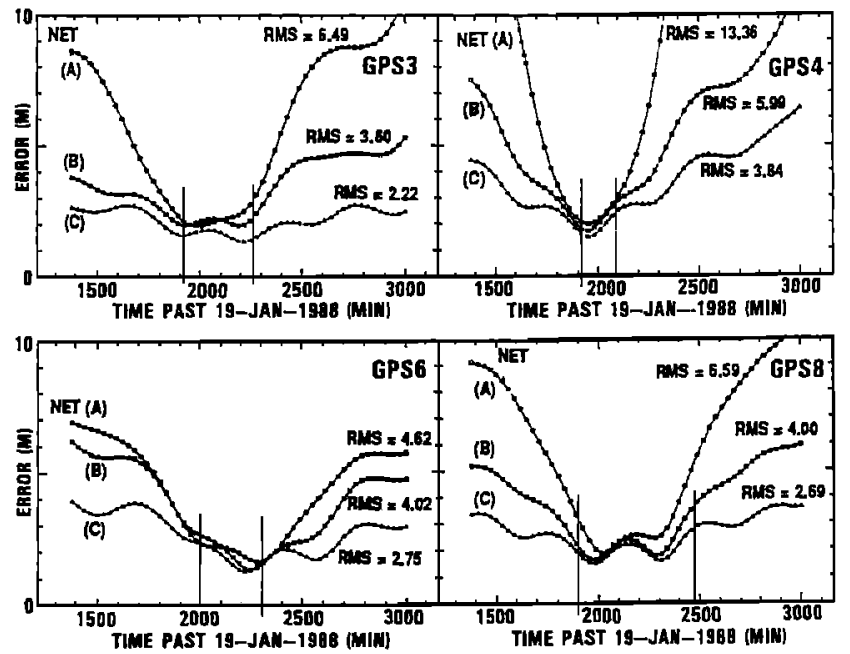

Fig. 3. Root mean square (ms) of the formal errors of the three orbit components (down track, cross track and radial) propagated in time for a single-day arc for networks A (U.S. only), B (U.S. and southwest Pacific) and C, the global tracking network (U.S., southwest Pacific and Europe) for GPS satellites $3,4,6$ and 8 . Lines indicate the time when the satellites converge over the CASA region.

Two stations, one on Hawaii and one on American Samoa, were also occupied as part of the global tracking network in the CASA Uno experiment. We analyzed the case where network (C) was supplemented by these two stations, but no significant improvement in the baseline estimates was seen. Preliminary analysis suggests that data noise from the Hawaiian station is large ( $\sim 4$ meter rms residial scatter on Pcode pseudorange compared to -1-2 meter scatter at other sites used in this analysis). Perhaps data from this station did not contribute to an already strong tracking network (C).

The improvements in GPS orbit and baseline estimates described in this paper were obtained with only 1 meter a priori spatial constraints on the non-U.S. global tracking stations. Presumably further improvement in precision and accuracy of CASA baseline estimates could be achieved if more of the global station positions were known at the centimeter level and could be held fixed or estimated with tighter positional constraints.

\section{Conclusions}

1) Results from the CASA Uno experiment confirm previous covariance studies showing that addition of global tracking sites, outside the regional network, to a nominal U.S. fiducial network can significantly improve baseline precision (Wu et al., 1988). East baseline component repeatability improved by a factor of 2.6 when two southwest Pacific sites and two European stations supplemented a U.S.-only network, while the north baseline component repeatability improved by a factor of 1.4 .

2) Using this global tracking network, short-term repeatability of horizontal baseline components better than 1 $\mathrm{cm}$ plus 3 parts in $10^{8}$ was achieved in the CASA region for baselines up to $1000 \mathrm{~km}$ in length.

3) Using the ability to resolve carrier phase cycle ambiguities for various network configurations as an indicator of GPS baseline accuracy improvement, we infer that this global tracking network improved the accuracy of the baseline estimates relative to the two smaller networks.

4) Improvements in baseline repeatability and accuracy with this global tracking network can be attributed to improved GPS satellite orbit estimates, a consequence of a global tracking network geometry.
Acknowledgements. This research was carried out by the Jet Propulsion Laboratory, California Institute of Technology, under contract with the National Aeronautics and Space Administration. We thank the many CASA Uno collaborators, particularly Ruth Neilan and Jim Kellogg. Geoff Blewith Steve Lichten, Tom Yunck and two anonymous reviewers provided helpful comments on the text.

\section{References}

Blewitt, G., Carrier Phase ambiguity resolution for the Globel Positioning System applied to geodetic baseline up to 2000 km, J, Geophys. Res., 94, 10187-10203, 1989.

Bock, Y., et al., A demonstration of 1-2 parts in 107 accuracy using GPS, Bull. Geod., 60, 241-254, 1986.

Davis, J. L., et al., Assessment of Global Positioning System measurements for studies of crustal deformation, J. Geophys. Res., 94, 13635-13650, 1989.

Dixon, T. H., et al., Constraints on Pacific plate kinematics and dynamics with Global Positioning System measurements, IEEE Trans. Geoscience and Remote Sensing, GE-23, No. 4, 491-501, 1985.

Dixon, T. H., S. Kornreich Wolf, Some tests of wet tropospheric calibration for the CASA Uno Global Positioning System Experiment, Geophys, Res, Lett. (IB Press), 1990.

Dong, D. and Y. Bock, Global Positioning System network analysis with phase ambiguity resolution applied to crustal deformation studies in California, J. Geophys. Res. 94, 3949-3966, 1989.

Herring, T. A., Precision of vertical position estimates for Very Long Baseline Interferometry, I. Geophys. Res. 91 , 9177-9182, 1986.

Kellogg, J., et al., CASA: Central and South America GPS geodesy, EOS: Trans. Am. Geophys. Union.70, 649-656, 1989.

Lichten, S. M., and J. S. Border, Strategies for high precision Global Positioning System orbit determination, $I$ Geophys. Res. 92, 12751-12762, 1987.

Lichten, S. M., High accuracy Global Positioning System orbit determination: Progress and prospects, Proceedings of Symposium \#102 of the International Association of Geodesy. General Meeting, (by Springer Verlag, ed. Y. Bock), In Press, 1990.

Mann, P., and K. Burke, Neatectonics of the Caribbean, Rev. Geophys, and Space Phys, 22,309-362, 1984.

Sovers, O. J., and J. S. Border, Observation model and parameter partials for JPL geodetic modeling software, "GPSOMC", JPL Pub. 87-21, Jet Propulsion Lab., Pasadena, CA., 1987.

Stein, S., et al., A test of alternative Caribbean plate motion models, J. Geophys. Res., 93, 3041-3050, 1988.

Tralli, D. M., et al., The effect of wet tropospheric path delays on estimation of geodetic baselines in the Gulf of California using the Global Positioning System, J. Geophys. Res..93, 6545-6557, 1988.

Wu, S. C., et al., Impact of tracking network variation on GPS orbit determination, paper AIAA-88-0573. AIAA 26th Aerospace Sciences Meeting, 1988.

T. H. Dixon and S. Kornreich Wolf, Jet Propulsioe Laboratory, mail code 183-501, California Institute of Technology, Pasadena, CA 91109.

J. T. Freymueller, Dept. of Geological Sciences, University of South Carolina, Columbia, SC 29208.

(Received November 14, 1989; revised February 9, 1990; accepted February 9, 1990.) 